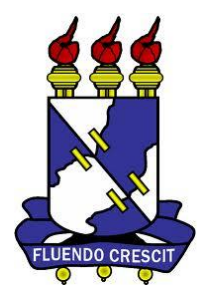

UNIVERSIDADE FEDERAL DE SERGIPE PRÓ-REITORIA DE PÓS-GRADUAÇÃO E PESQUISA MESTRADO EM CIÊNCIAS DA SAÚDE

MARCOS GABRIEL DO NASCIMENTO JUNIOR

AVALIAÇÃO DA DPOC EM TABAGISTAS COM SUSPEITA DE ISQUEMIA MIOCÁRDICA 
MARCOS GABRIEL DO NASCIMENTO JUNIOR

\section{AVALIAÇÃO DA DPOC EM TABAGISTAS COM SUSPEITA DE ISQUEMIA MIOCÁRDICA}

Dissertação apresentada ao Programa de PósGraduação em Ciências da Saúde da Universidade Federal de Sergipe como requisito parcial à obtenção do título de Mestre em Ciências da Saúde.

Orientadora: Prof. ${ }^{a}$ Dr. ${ }^{a}$ Maria Luiza Dória Almeida

ARACAJU

2017 


\section{FICHA CATALOGRÁFICA ELABORADA PELA BIBLIOTECA BISAU UNIVERSIDADE FEDERAL DE SERGIPE}

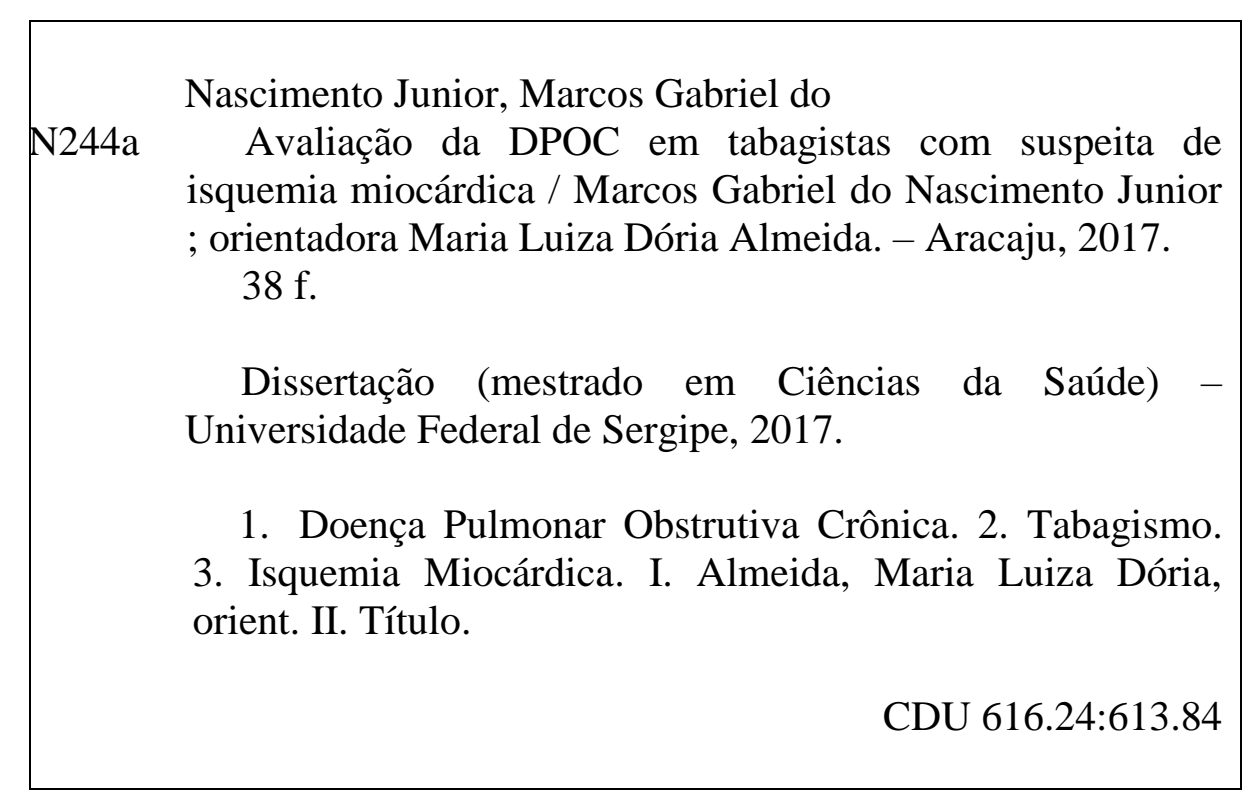


MARCOS GABRIEL DO NASCIMENTO JUNIOR

\section{AVALIAÇÃO DA DPOC EM TABAGISTAS COM SUSPEITA DE ISQUEMIA MIOCÁRDICA}

Dissertação apresentada ao Programa de PósGraduação em Ciências da Saúde da Universidade Federal de Sergipe como requisito parcial à obtenção do título de Mestre em Ciências da Saúde.

Aprovada em:

Prof. ${ }^{a}$ Dr. ${ }^{a}$ Maria Luiza Dória Almeida Presidente

Prof. Dr. José Barreto Neto

$1^{\circ}$ Examinador

Prof. Dr. Aquiles Assunção Camelier

$\mathbf{2}^{\circ}$ Examinador 


\section{RESUMO}

Introdução: A Doença Pulmonar Obstrutiva Crônica (DPOC) é uma das principais causas de morbimortalidade no adulto acima de 40 anos, sendo caracterizada por limitação do fluxo aéreo e inflamação, com diversas manifestações sistêmicas. Sua principal comorbidade é a doença cardiovascular, responsável por alto impacto no prognóstico e mortalidade do portador dessa doença. $\mathrm{O}$ objetivo desta pesquisa foi comparar indivíduos tabagistas com e sem DPOC, requeridos por suspeita de isquemia miocárdica. Métodos: A população do estudo foi composta por indivíduos submetidos à Ecocardiografia sob Estresse pelo Esforço Físico (EEEF) para diagnóstico de isquemia miocárdica, investigados clinicamente e submetidos a exame de função pulmonar para diagnóstico da DPOC. Foram avaliados 267 tabagistas reunidos em grupo G1: os que preenchiam diagnóstico de DPOC com 121 participantes (45\%), sendo 63,6\% portadores da DPOC leve, segundo critério do GOLD (2017); e grupo G2: sem DPOC com 146 participantes (55\%). Resultados: A presença de isquemia miocárdica foi significativamente diferente entre os grupos $(\mathrm{p}<0,001)$, independentemente das variáveis comuns aos tabagistas e da classificação da doença. Estatisticamente importante também foi a presença das comorbidades no grupo G1 (osteoporose, depressão e neoplasias). Conclui-se que em indivíduos com suspeita de isquemia miocárdica, a investigação da DPOC é fundamental, pois sua presença é estatisticamente maior nos tabagistas com critérios da DPOC, mesmo em fase inicial.

Descritores: Doença Pulmonar Obstrutiva Crônica. Tabagismo. Isquemia Miocárdica. 


\begin{abstract}
Introduction: Chronic Obstructive Pulmonary Disease (COPD) is one of the main causes of morbidity and mortality in adults over 40 years old, characterized by airflow limitation and inflammation, with several systemic manifestations. Its main comorbidity is cardiovascular disease, which is responsible for a high impact on the prognosis and mortality of patients with this disease. The objective of this research was to compare smokers with and without COPD, required for suspicion of myocardial ischemia. Methods: The study population consisted of individuals submitted to Physical Stress Echocardiography (EEEF) for the diagnosis of myocardial ischemia, clinically investigated and submitted to pulmonary function examination to diagnose COPD. A total of 267 smokers were enrolled in a G1 group: those with a diagnosis of COPD with 121 participants (45\%), 63.6\% with mild COPD, according to GOLD criteria (2017); And group G2: without COPD with 146 participants (55\%).Results: The presence of myocardial ischemia was significantly different between the groups $(\mathrm{p}<0.001)$, independently of the variables common to smokers and the classification of the disease. Statistically important was also the presence of comorbidities in the G1 group (osteoporosis, depression and neoplasms). It is concluded that in individuals with suspected myocardial ischemia, the investigation of COPD is fundamental, since its presence is statistically higher in smokers with COPD criteria, even in the initial phase.
\end{abstract}

Keywords: Chronic Obstructive Pulmonary Disease. Smoker. Myocardial Ischemia. 


\section{LISTA DE ABREVIATURAS}

AE: Átrio Esquerdo

CAT: Chronic Obstructive Pulmonary Disease Assessment Test

CVF: Capacidade Vital Forçada

DM: Diabetes Melito

DPOC: Doença Pulmonar Obstrutiva Crônica

EEEF: Ecocardiografia sob Estresse pelo Esforço Físico

FC: Frequência Cardíaca

FEF 25-75\%: Fluxo Expiratório Forçado entre 25 e $75 \%$

GOLD: Global Initiative for Chronic Obstructive Lung Disease

HAS: Hipertensão Arterial Sistêmica

IAM: Infarto Agudo do Miocárdio

IMC: Índice de Massa Corpórea

mMRC: Medical Research Council modificado

Relação E/E': Combinação da velocidade diastólica precoce do fluxo mitral (E) e a velocidade do anel (E')

VE: Ventrículo Esquerdo

VEF1: Volume Expiratório Forçado no primeiro segundo

VEF1/CVF: Relação entre o Volume Expiratório Forçado no primeiro segundo e a Capacidade Vital Forçada 


\section{SUMÁRIO}

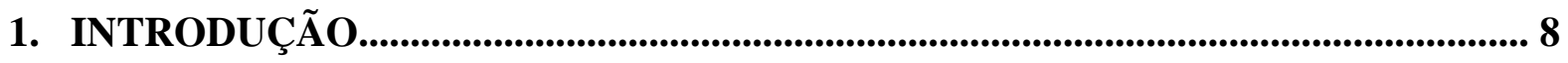

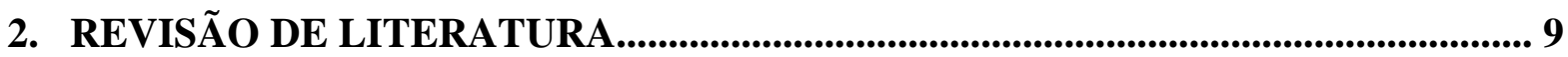

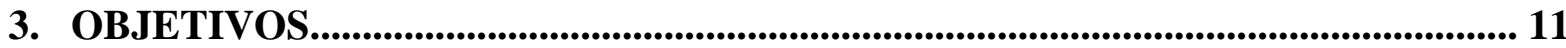

3.1 Objetivo Geral.......................................................................................................................11

3.2 Objetivo Específico.......................................................................................................................11

4. CASUÍSTICA E MÉTODOS.......................................................................................12

4.1 Caracterização do estudo..................................................................................................12

4.2 Local e Período..................................................................................................................................12

4.3 População e Amostra............................................................................................................12

4.4 Questões Éticas...........................................................................................13

4.5 Procedimento Experimental................................................................................................13

4.5.1 Procedimentos e instrumentos da avaliação ecocardiográfica.........................13

4.5.2 Procedimentos e instrumentos de avaliação da DPOC....................................14

4.6 Análise e Interpretação de resultados..................................................................................14

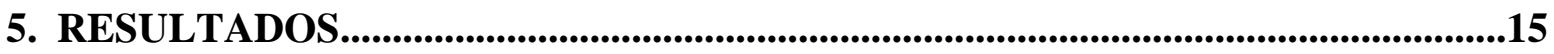

5.1 Dados antropométricos e sociodemográficos..................................................................15

5.2 Fatores de risco: exposição e intensidade.....................................................................16

5.3 Resultados da EEEF............................................................................................................17

5.4 Achados estruturais e funcionais da EEEF.................................................................17

5.5 Sintomas, escala de dispneia e comorbidades...................................................................18

5.6 Sintomas e fatores de risco para isquemia..............................................................19

5.7 Gravidade da DPOC (GOLD) ..........................................................................................20

5.8 Regressão Logística...................................................................................................................21

6. DISCUSSÃO

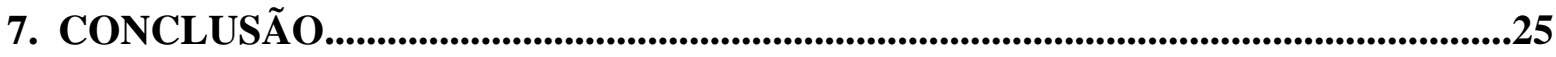

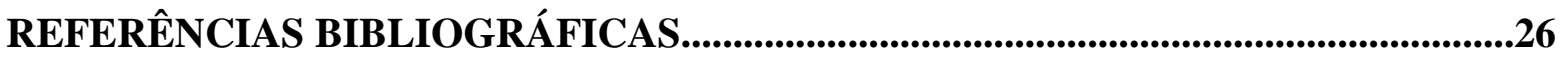

APÊNDICE....................................................................................................................................31

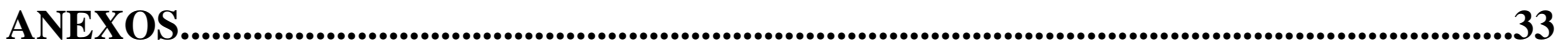




\section{INTRODUÇÃO}

A Doença Pulmonar Obstrutiva Crônica (DPOC) é uma doença comum, prevenível e tratável, caracterizada por sintomas respiratórios persistentes e limitação ao fluxo aéreo, devido a alterações nas vias aéreas e nos alvéolos, causadas por exposições significativas às partículas e gases nocivos, sendo o tabagismo o fator que se coloca como principal causa geradora desta afecção (GOLD, 2017; NEVES et al., 2013).

A DPOC é uma das principais causas de morte e custos para saúde, além de ser a maior causa de morbidade no mundo. Entre $10 \%$ e $20 \%$ da população dos fumantes com mais de 40 anos são portadores de DPOC, caracterizada por ser uma doença lenta e progressiva que diminui o fluxo aéreo, ocasionando perda funcional pulmonar (BHANDARI; SHARMA, 2012; HAN et al., 2010; SHAVELLE et al., 2009).

Apesar de ser uma doença de caráter respiratório, a DPOC traz consigo vários prejuízos no âmbito sistêmico. As manifestações sistêmicas se acentuam nos períodos de exacerbação da DPOC, o que acaba constituindo um fator importante na progressão da perda da função pulmonar, resultando assim em uma piora da qualidade de vida (LINS; ESTEVES; VIDIGAL, 2012; PRICE et al., 2011).

O aumento do tabagismo em países em desenvolvimento é responsável por mais de $50 \%$ das mortes por neoplasias, sendo, dessas, $95 \%$ de pulmão. Ele também é culpado por $73 \%$ das mortes por DPOC e $20 \%$ por doenças cardiovasculares, fator que o coloca como primordial nas políticas de saúde pública. Estima-se que 1,2 bilhões de pessoas no mundo sejam fumantes, segundo a Organização Mundial da Saúde (OMS). Outro fato importante é que cerca de 5\% de toda população americana tem DPOC. No Brasil, existem 40.000 mortes por DPOC ao ano (LINS; ESTEVES; VIDIGAL, 2012; SILVA et al., 2014; WORLD HEALTH ORGANIZATION, 2014).

As associações entre a DPOC e suas comorbidades são explicadas de duas formas, a primeira demonstra que são resultados de diversos eventos inflamatórios e reparatórios, que ocorrem nos pulmões destes pacientes, sendo este o centro do processo. E a segunda demonstra que as manifestações pulmonares do DPOC têm um caráter inflamatório de âmbito mais sistêmico, com comprometimento de vários órgãos e funções. Revisões recentes já aceitam que essas manifestações ocorrem de uma forma simultânea (BARNES; CELLI, 2009). 
Portadores da DPOC possuem maior prevalência de Hipertensão Arterial Sistêmica (HAS), Diabetes Melito (DM) e dislipidemia, fatores que estão relacionados diretamente ao risco de isquemia miocárdica. Ressaltando que comprometimento cardiovascular é a principal comorbidade da DPOC, a Ecocardiografia sob Estresse pelo Esforço Físico (EEEF) poderá ser utilizada para a detecção da isquemia miocárdica (ANDRADE et al., 2002; MESQUITA; JORGE, 2013; SCHETTINO et al., 2013).

Partindo da premissa do binômio DPOC e isquemia miocárdica, com eventos integrados e de relevante importância para o desfecho prognóstico da DPOC, além da escassez de estudos utilizando o estresse físico como indutor da isquemia no portador de obstrução pulmonar, esta pesquisa foi delineada para avaliar a presença da DPOC em indivíduos com suspeita de isquemia miocárdica, pretendendo contribuir para esclarecimento desta associação.

\section{REVISÃO DE LITERATURA}

A DPOC tem um perfil que está diretamente relacionado com o tabaco, que possui mais de 4.700 compostos de substâncias químicas nocivas ao corpo, radicais livres e outros oxidantes presentes em altas concentrações. A DPOC representa a $4^{\mathrm{a}}$ maior causa de morte do mundo e prevê-se que seja a $3^{\mathrm{a}}$ até 2030. No Brasil entre 3 e 7 milhões de pessoas são portadoras da DPOC, o estudo Projeto Latino-Americano de Investigação em Obstrução Pulmonar (Platino) demonstrou uma prevalência de 15,8\% nos adultos acima de 40 anos, na cidade de São Paulo (ALEXOPOULOS et al., 2015; LOPEZ et al., 2006; MENEZES, 2006; SCHNEIDERS et al., 2015).

No mundo o tabagismo é responsável por uma morte em cada dez indivíduos. Um dado relevante é que em 2012 morreram 3,1 milhões de pessoas devido à DPOC. O tabagismo com uso contínuo determina mais de 50\% de obstrução da via aérea, em indivíduos com mais de 70 anos (DIAZ-GUZMAN; MANNINO, 2014; PESSÔA; PESSÔA, 2009; WORLD HEALTH ORGANIZATION, 2014).

O número de pessoas que se declaram fumantes cresce no mundo, estudos indicam que existirá cerca de 2 bilhões de fumantes no ano de 2030, tendo sua maior concentração nos países em desenvolvimento. A temática, no Brasil, já é abordada com frequência há algum tempo, onde campanhas sobre os males do uso do tabaco figuram entre os orçamentos públicos, leis de 
proibição foram implantadas, porém os gastos do Sistema Único de Saúde têm aumentado para o tratamento das doenças pulmonares decorrentes do tabaco, dentre as quais a DPOC (WÜNSCH FILHO et al., 2010).

As mortes por DPOC ocorrem em $90 \%$ dos casos nos países subdesenvolvidos, pois são desprovidos de recursos para saúde. Outros fatores que contribuem são: a dificuldade para realização do diagnóstico, através da espirometria, assim como a carência de profissionais capacitados. Em contraste com a situação, os Estados Unidos gastaram, em 2010, cerca de US\$ 49,9 bilhões, com pacientes portadores de DPOC, demonstrando a relevância da doença, que se encontra em ascensão (ADELOYE et al., 2015; WORLD HEALTH ORGANIZATION, 2014).

A incidência da DPOC pelo mundo varia de acordo com a quantidade do uso do tabaco e também com a expectativa de vida de cada população. O estudo de Pessôa e Pessôa (2009) utilizou uma amostra de 1.897 indivíduos que realizaram testes de função pulmonar constatando que o aumento da idade influenciava em apenas $0,7 \%$ na incidência de obstrução do fluxo aéreo em não tabagistas, demonstrando assim que o tabaco se apresenta como o principal causador da DPOC (HAGSTAD et al., 2012).

As comorbidades que ocorrem em indivíduos com DPOC são diversas, se destacam, entretanto, as que possuem impacto na mortalidade. Neste contexto, as doenças cardiovasculares são as principais, seguidas pela depressão, osteoporose e neoplasia de pulmão. Todas essas comorbidades têm relação com um prognóstico ruim (MAHBOUB et al., 2016; ONGEL et al., 2014).

Comorbidades são determinantes para demonstração da qualidade de vida dos pacientes, quanto mais elas existem e estão associadas, pior será a qualidade de vida do portador. Além das comorbidades mais conhecidas, a DPOC ainda apresenta outras não tão frequentes, como: insônia, doenças locomotoras, sinusite, enxaqueca, úlceras de estômago e duodeno, angina, catarata, pneumonia e outras infecções respiratórias (SORIANO et al., 2005).

As comorbidades são ponderadas como a principal causa de morte, principalmente as cardiovasculares. O diagnóstico das comorbidades pode resultar em intervenção precoce, para que possa existir possibilidade de tratamento, podendo gerar melhor qualidade de vida, além de redução da mortalidade. Intervenções preventivas, do ponto de vista cardiovascular, podem levar a ajustes das necessidades e cuidados básicos dos pacientes. (DIVO et al., 2012; FEARY et al., 2010; STÄLLBERG et al., 2016). 
A DPOC tem efeitos consideráveis sobre o sistema cardiovascular. Pesquisas demonstram que $20 \%$ dos pacientes com DPOC têm algum tipo de comorbidade cardiovascular diagnosticada; destacam-se: cardiopatias isquêmicas, arritmias, que são sempre associadas à redução da sobrevida dos portadores da DPOC (GUPTA; MANN, 2016; MACDONALD et al., 2016).

A Ecocardiografia constitui importante subsídio, tanto para elucidação diagnóstica, como para avaliação do prognóstico em pacientes com doença arterial coronariana. Como a Ecocardiografia possibilita a avaliação em tempo real da motilidade ventricular esquerda, técnicas que utilizam estresse pelo esforço físico ou farmacológico, seja ele inotrópico ou vaso dilatador, permitem a documentação da extensão e da gravidade das alterações transitórias da motilidade ventricular esquerda (CAMPOS FILHO et al., 2004).

Devido à sua acurácia, praticidade e boa relação custo-benefício, a Ecocardiografia tem sido vista com grande interesse. A EEEF é um método não invasivo, para determinação do diagnóstico e prognóstico, na avaliação do impacto de terapias de revascularização, na detecção de viabilidade miocárdica e no auxílio das decisões terapêuticas (BOCCHI et al., 2009; CÉSAR et al., 2004).

Pesquisas recentes sugerem que a isquemia miocárdica, durante as exacerbações da DPOC, é comum e presume mal prognóstico. Vale ressaltar que as exacerbações pulmonares e cardíacas acontecem de forma conjunta, porém esta correlação é de difícil detecção clínica (HASAN et al., 2014; MACDONALD et al., 2016).

\section{OBJETIVOS}

\subsection{Objetivo Geral}

Avaliar a DPOC em tabagistas com suspeita de isquemia miocárdica.

\subsection{Objetivos Específicos}

Diagnosticar a DPOC;

Comparar os achados funcionais e estruturais do EEEF;

Analisar as comorbidades do DPOC. 


\section{CASUÍSTICA E MÉTODOS}

\subsection{Caracterização do Estudo}

Trata-se de um estudo analítico, transversal de abordagem quantitativa.

\subsection{Local e período}

A pesquisa foi realizada na cidade de Aracaju/Sergipe, na Fundação São Lucas, onde funciona o laboratório de Ecocardiografia (Ecolab). A pesquisa faz parte dos trabalhos desenvolvidos no Programa de Pós-Graduação em Ciências da Saúde da Universidade Federal de Sergipe (UFS), no período de fevereiro de 2015 a dezembro de 2016.

\subsection{População e Amostra}

A amostra foi obtida por conveniência, composta por indivíduos com mais de 40 anos, que eram tabagistas, tendo como critério possuir carga tabágica de no mínimo um maço/ano, além da indicação de um médico assistente para realizar a EEEF, e completarem a realização do exame.

Esses pacientes selecionados foram divididos em dois grupos: Grupo Um (G1): tabagistas que apresentaram diagnóstico de DPOC; e o Grupo Dois (G2): tabagistas que não apresentaram diagnóstico de DPOC. O cálculo do tamanho de amostra para pesquisa em Ciências da Saúde pode ser estimado em proporção (LWANGA; LEMESHOW, 1991); baseando-se na última estimativa de tabagismo do Ministério da Saúde para Sergipe (MS, 2015), a prevalência do hábito de fumar em Aracaju é de 10,6\% em adultos. Assim, utilizando o método da estimação de uma proporção, um nível de confiança de $95 \%$ e um erro máximo desejado de 5\%, a amostra proposta por esta pesquisa teria de ser acima de 138 indivíduos tabagistas. Porém, para que o trabalho tivesse uma maior relevância científica essa amostra foi aumentada, sendo composta por 267 indivíduos, dos quais 121 com diagnóstico de DPOC e 146 sem.

Foram excluídos da amostra os pacientes portadores de asma, doença ocupacional e outras patologias pulmonares como: sequelas de tuberculose pulmonar e/ou fibrose pulmonar que atrapalhassem o diagnóstico de DPOC. 


\subsection{Questões Éticas}

A pesquisa atendeu às normas éticas exigidas pela Resolução 466/2012 (Conselho Nacional de Saúde), sendo submetida ao Comitê de Ética em Pesquisa da UFS. Com este trabalho fazendo parte da extensão do projeto Doença Pulmonar Obstrutiva Crônica: interação e efeitos sob as cardiopatias, aprovado sob o CAAE 06181712.9.0000.5546. Além disso, todos os pacientes receberam o Termo de Consentimento Livre e Esclarecido (Apêndice) por escrito, depois de receberem os esclarecimentos sobre o estudo.

\subsection{Procedimento experimental}

Primeiramente os pacientes realizavam a avaliação clínica (Anexo A), seguido pela EEEF e logo depois eram aplicados os testes clínicos da DPOC (Anexos B e C), finalizando com a realização da espirometria (pré e pós-broncodilatador).

\subsubsection{Procedimentos e instrumentos da avaliação ecocardiográfica}

Todos os pacientes foram submetidos ao exame ecocardiográfico observando-se os aspectos técnicos do exame, seguindo a Sociedade Americana de Ecocardiografia, o aparelho utilizado foi o Phillips IE-33 (LANG et al., 2015).

Todas as imagens assim obtidas foram selecionadas, dispostas lado a lado em formato quádruplo, para análise de forma comparativa, com diferentes níveis de frequência cardíaca (FC), por um ecocardiografista experiente, possuidor de nível III, por avaliação específica.

O espessamento parietal segmentar do ventrículo esquerdo (VE) foi avaliado, quantitativamente, com a utilização do modelo de 16 segmentos e graduado em: 1-normal, 2hipocinético, 3- acinético e 4- discinético. De acordo com o índice de escore da motilidade miocárdica do VE (IEMVE), os pacientes eram classificados como normais (igual a 1), com disfunção ventricular leve (entre 1,01 - 1,60), com disfunção ventricular moderada (entre 1,6 2,0 ) e com disfunção ventricular grave (maior que 2,0). O desenvolvimento de alterações de motilidade segmentar induzidas pelo esforço poderá ser considerado como indicador de isquemia miocárdica (GARDIN et al., 2002). 


\subsubsection{Procedimentos e instrumentos de avaliação da DPOC}

Todos os indivíduos realizaram testes espirométricos pré e pós-broncodilatador, sob a responsabilidade de um pneumologista e seguindo as normas da American Thoracic Society (NAKAJIMA et al., 2008), sendo obtidas as medidas de Volume Expiratório Forçado no primeiro segundo (VEF1), Capacidade Vital Forçada (CVF), a relação VEF1/CVF e o Fluxo Expiratório Forçado Intermediário (FEF 25 - 75\%).

Quando a relação do VEF1/CVF foi menor que 70\%, após o uso do broncodilatador, o diagnóstico de limitação ao fluxo aéreo ficava definido, característica da DPOC (GOLD, 2017).

As espirometrias foram realizadas com o aparelho Microloop modelo MK8 com Software Spida 05, o padrão utilizado foi o de Pereira (2002) e os testes pós-broncodilatador foram avaliados após a inalação de salbutamol (400 microgramas).

Além da espirometria, a avaliação seguiu as recomendações do GOLD (DUSSER et al., 2016; GOLD, 2017) classificando os estágios de gravidade da DPOC (I, II, II e IV). Para classificação da DPOC foram aplicados os seguintes questionários:

- A Escala de dispneia modificada do Medical Research Council (mMRC) (ANEXO B), avalia a sensação de desconforto respiratório (KOVELIS et al., 2008).

- O Teste do CAT (COPD Assessment Test - CAT), (ANEXO C), foi utilizado para medir o impacto da DPOC no bem-estar e no quotidiano dos doentes. É uma mensuração simples e confiável do estado geral de saúde relacionado à DPOC para a avaliação e acompanhamento de pacientes individuais (JONES et al., 2009).

\subsection{Análise e Interpretação dos Resultados}

A análise descritiva foi realizada através de medidas de tendência central e variabilidade. No caso das variáveis numéricas, foram utilizadas média e desvio padrão quando os dados foram considerados normais, a mediana e amplitude interquartil quando os dados tiveram uma distribuição considerada assimétrica. Com relação às variáveis categóricas, foi utilizada frequência absoluta e relativa. A simetria da distribuição das variáveis quantitativas foi analisada através do teste Shapiro-Wilk. Para análise das variáveis categóricas foi realizado o teste do QuiQuadrado; as variáveis numéricas foram avaliadas através do teste Mann-Whitney, quando 
assimétricas, e através do teste t de Student, quando simétricas. O nível de significância adotado foi de 5\%. A associação entre a ocorrência de isquemia miocárdica à EEEF e os fatores de risco clássicos para isquemia, incluindo a DPOC, foram avaliados mediante regressão logística.

\section{RESULTADOS}

\subsection{Dados antropométricos e sociodemográficos}

Foram avaliados 267 pacientes de ambos os sexos, os quais foram divididos em Grupo Tabagista com DPOC (G1) com 121 participantes (45\%) e Grupo Tabagista sem DPOC (G2) com 146 participantes $(55 \%)$.

$\mathrm{Na}$ distribuição dos grupos o G1 teve idade maior $(\mathrm{p}=0,012)$, não houve diferença significativa em altura, peso e consequentemente o Índice de Massa Corpórea (IMC). Não houve diferença significativa entre sexo, cor observada e a escolaridade. Os homens representaram 60\% da amostra e as mulheres 40\%, para ambos os grupos. A distribuição de cor observada foi G1 (69 e $31 \%$ ) e G2 (73 e 27\%), classificados como brancos e negros/pardos respectivamente. 
Tabela 1 - Características antropométricas e sociodemográficas

\begin{tabular}{lrrr}
\hline & G1 & G2 & Valor de p \\
\hline Idade (anos) & $\mathbf{6 2 , 4 2} \pm \mathbf{9 . 3 4}$ & $\mathbf{5 9 , 3 3} \pm \mathbf{9 . 3 9}$ & $\mathbf{0 , 0 1 2}$ \\
Altura (cm) & $164,15 \pm 8.84$ & $164,69 \pm 9.10$ & 0,550 \\
Peso (kg) & $73,02 \pm 14.90$ & $75,86 \pm 14.14$ & 0,052 \\
Índice de Massa Corpórea & $27,03 \pm 4.66$ & $27,82 \pm 4.12$ & 0,073 \\
Sexo & & & 1,000 \\
Masculino & $73(60 \%)$ & $88(60 \%)$ & \\
Feminino & $48(40 \%)$ & $58(40 \%)$ & 0,679 \\
Escolaridade & & & \\
Analfabeto & $4(3 \%)$ & $5(3 \%)$ & \\
$1^{\circ}$ Grau & $22(18 \%)$ & $65(14 \%)$ & \\
$2^{\circ}$ Grau & $55(45 \%)$ & $44(30 \%)$ & \\
$3^{\circ}$ Grau & $28(23 \%)$ & $12(8 \%)$ & \\
Pós-Graduação* & $12(10 \%)$ & $107(73 \%)$ & \\
Cor observada & & $39(27 \%)$ & \\
Branco & $84(69 \%)$ & $37(31 \%)$ & \\
Negro/Pardo & & & \\
\hline
\end{tabular}

* Pós-graduação: pós-graduados, mestres e doutores.

Fonte: Tabela elaborada pelo autor.

\subsection{Fatores de risco: exposição e intensidade}

Houve diferença significativa entre G1 e G2 com relação ao hábito tabágico, com 44\% de tabagistas ativos no G1 e apenas $18 \%$ de tabagistas ativos no G2. Com relação ao tempo de tabagismo existiu diferença, sendo o grupo G1 com maior tempo de tabagismo, logo também diferiram quanto ao número de quantidade de maços/ano. Também existiu diferença significativa sobre exposição à lenha, não havendo com relação à ocupação de risco. 
Tabela 2 - Fatores de risco: exposição e intensidade

G1

G2

Valor $\mathbf{p}$

Hábito Tabágico

Tabagista

Ex-tabagista

Tempo de Tabagismo (anos)

Quantidade de maços/ano

Exposição à lenha

Ocupação de risco**
$53(44 \%)$

$68(56 \%)$

$24,28 \pm 12,57$

$26,06 \pm 28,72$

Mediana

20,00*

$6(5 \%)$

$3(2 \%)$

$27(18 \%)$

$<0,001$

$119(82 \%)$

$20,35 \pm 13,40$

$\mathbf{0 , 0 0 7}$

$20,30 \pm 24,65$

$\mathbf{0 , 0 0 8}$

Mediana 20,00*

$\mathbf{0 , 0 0 7}$

* Mediana para valores em que o desvio padrão foi maior que a média.

** Ocupação de risco: trabalhadores de indústrias químicas, sílica.

Fonte: Tabela elaborada pelo autor.

\subsection{Resultados da EEEF}

$\mathrm{O}$ resultado da EEEF na comparação entre grupos foi significativamente diferente ( $\mathrm{p}<$ 0,001), com $61 \%$ de EEEF positivos no G1.

Tabela 3 - Resultados EEEF

\begin{tabular}{lrrrrrr}
\hline & \multicolumn{2}{c}{ G1 } & \multicolumn{2}{c}{ G2 } & Total & Valor de p \\
\hline Resultado EEEF & N & \% & N & \% & & \\
\hline Positivo & $\mathbf{7 4}$ & $\mathbf{6 1 \%}$ & $\mathbf{3 7}$ & $\mathbf{2 5 \%}$ & $\mathbf{1 1 1}$ & $<\mathbf{0 , 0 0 1}$ \\
Negativo & 47 & $39 \%$ & 109 & $75 \%$ & 156 & \\
Total & 121 & $100 \%$ & 146 & $100 \%$ & 267 & \\
\hline
\end{tabular}

* EEEF - Ecocardiografia sob Estresse pelo Esforço Físico.

Fonte: Tabela elaborada pelo autor.

\subsection{Achados estruturais e funcionais da EEEF}

Existiu diferença significativa nos valores de índice de escore de motilidade do ventrículo esquerdo, tempo em esteira, volume de oxigênio máximo, estimativa do equivalente metabólico avaliado, frequência cardíaca máxima e índice de massa do ventrículo esquerdo. 
Tabela 4 - Achados estruturais e funcionais da EEEF em pacientes tabagistas

\begin{tabular}{lrrrrr}
\hline & \multicolumn{2}{c}{ G1 } & G2 & Valor de p \\
\hline & Média & Desvio & Média & Desvio & \\
& & Padrão & & Padrão & \\
\hline IEMVE* & $\mathbf{1 , 0 8}$ & $\mathbf{0 , 1 2}$ & $\mathbf{1 , 0 3}$ & $\mathbf{0 , 0 6}$ & $<\mathbf{0 , 0 0 1}$ \\
Tempo em Esteira (min) & $\mathbf{6 , 2 9}$ & $\mathbf{1 , 9 5}$ & $\mathbf{7 , 1 9}$ & $\mathbf{2 , 1 2}$ & $<\mathbf{0 , 0 0 1}$ \\
VO2 máx*** (ml/kg/min) & $\mathbf{3 3 , 3 1}$ & $\mathbf{9 , 1 3}$ & $\mathbf{3 7 , 1 7}$ & $\mathbf{8 , 6 9}$ & $<\mathbf{0 , 0 0 1}$ \\
MET*** avaliado & $\mathbf{9 , 5 9}$ & $\mathbf{2 , 5 1}$ & $\mathbf{1 0 , 5 9}$ & $\mathbf{2 , 5 4}$ & $\mathbf{0 , 0 0 1}$ \\
MET*** previsto & 7,61 & 1,58 & 7,87 & 1,84 & 0,310 \\
FC máxima & $\mathbf{1 4 8 , 1 9}$ & $\mathbf{2 3 , 8 3}$ & $\mathbf{1 5 1 , 7 1}$ & $\mathbf{1 9 , 3 6}$ & $\mathbf{0 , 0 3 1}$ \\
Diâmetro Aorta & 3,28 & 0,39 & 3,32 & 0,40 & 0,571 \\
Fração de Ejeção & 0,68 & 0,10 & 0,69 & 0,08 & 0,609 \\
Espessura Relativa & 31,03 & 3,91 & 31,45 & 4,13 & 0,475 \\
Átrio esquerdo & 3,86 & 0,63 & 3,87 & 0,43 & 0,304 \\
Diâmetro Sistólico VE (cm) & 3,24 & 0,67 & 3,19 & 0,53 & 0,981 \\
Diâmetro Diastólico VE & 5,27 & 0,62 & 5,24 & 0,56 & 0,924 \\
Parede Posterior (cm) & 0,81 & 0,09 & 0,82 & 0,10 & 0,520 \\
Índice de Massa VE & $\mathbf{9 0 , 1 8}$ & $\mathbf{2 1 , 8 4}$ & $\mathbf{8 3 , 5 1}$ & $\mathbf{1 9 , 7 5}$ & $\mathbf{0 , 0 0 3}$ \\
Volume Indexado do AE & 21,94 & 9,19 & 22,10 & 6,43 & 0,239 \\
Relação E/E' & 10,47 & 3,50 & 10,14 & 3,09 & 0,595 \\
\hline * Índice do Escore de Motilidade Ventricular Esquerda (IEMVE). & & & \\
** Volume de Oxigênio Máximo (VO2 máx). & & & & & \\
*** Estimativa do equivalente metabólico (MET). & & & & & \\
Fonte: Tabela elaborada pelo autor. & & & & &
\end{tabular}

\subsection{Sintomas, escala de dispneia e comorbidades}

O grupo G1 apresentou maior presença de tosse e secreção. Sobre a escala de dispneia (mMRC), o G1 e o G2 apresentaram respectivamente 71\% e 47\% com mMRC 1, com diferença significativa. Comorbidades como: osteoporose, depressão e neoplasias aparecem com diferença significativa, sendo o G1 com maior frequência de comorbidades. 
Tabela 5 - Presença de sintomas, escala de dispneia e comorbidades

\begin{tabular}{|c|c|c|c|c|c|c|}
\hline & \multicolumn{2}{|c|}{ G1 } & \multicolumn{2}{|c|}{ G2 } & \multirow[t]{2}{*}{ Total } & \multirow[t]{2}{*}{ Valor $\mathbf{p}$} \\
\hline & $\mathbf{N}$ & $\%$ & $\mathbf{N}$ & $\%$ & & \\
\hline Tosse Crônica (CAT)* & 104 & $86 \%$ & 64 & $44 \%$ & 168 & $<0,001$ \\
\hline Expectoração Crônica (CAT)* & 96 & $79 \%$ & 66 & $45 \%$ & 162 & $<0,001$ \\
\hline \multicolumn{7}{|l|}{ Escala de Dispneia (mMRC)** } \\
\hline $\mathbf{0}$ & 8 & $7 \%$ & 58 & $40 \%$ & 66 & $<0,001$ \\
\hline 1 & 86 & $71 \%$ & 69 & $47 \%$ & 155 & \\
\hline 2 & 22 & $18 \%$ & 14 & $10 \%$ & 36 & \\
\hline 3 & 5 & $4 \%$ & 5 & $3 \%$ & 10 & \\
\hline 4 & $\mathbf{0}$ & $\mathbf{0 \%}$ & $\mathbf{0}$ & $\mathbf{0 \%}$ & $\mathbf{0}$ & \\
\hline Osteoporose & 52 & $43 \%$ & 16 & $11 \%$ & 68 & $<0,001$ \\
\hline Depressão & 48 & $40 \%$ & 22 & $15 \%$ & 70 & $<0,001$ \\
\hline Neoplasias & 31 & $26 \%$ & 5 & $3 \%$ & 36 & $<0,001$ \\
\hline
\end{tabular}

* Tosse e expectoração crônica pertencentes ao Escore CAT.

** mMRC Escala modificada Medical Research Council.

Fonte: Tabela elaborada pelo autor.

\subsection{Sintomas e fatores de risco para isquemia}

Existiu diferença significativa com relação aos resultados de cateterismos positivos, existência de infarto antigo e presença de revascularização. Sobre fatores de risco para isquemia, não apresentaram diferença significativa para: HAS, DM, dislipidemia, antecedentes familiares, atividade física e etilismo. 
Tabela 6 - Sintomas e fatores de risco para isquemia

\begin{tabular}{|c|c|c|c|c|c|c|}
\hline & \multicolumn{2}{|c|}{ G1 } & \multicolumn{2}{|c|}{ G2 } & \multirow[t]{2}{*}{ Total } & \multirow[t]{2}{*}{ Valor p } \\
\hline & $\mathbf{N}$ & $\%$ & $\mathbf{N}$ & $\%$ & & \\
\hline \multicolumn{7}{|c|}{ Aparecimento de Sintomas na EEEF* } \\
\hline Assintomático & 44 & $36 \%$ & 83 & $57 \%$ & 127 & $\mathbf{0 , 0 0 3}$ \\
\hline Precordialgia típica & 34 & $28 \%$ & 20 & $14 \%$ & 54 & \\
\hline Precordialgia atípica & 39 & $32 \%$ & 39 & $27 \%$ & 78 & \\
\hline Dispneia & 4 & $3 \%$ & 4 & $3 \%$ & 8 & \\
\hline \multicolumn{7}{|l|}{ Cateterismo } \\
\hline Positivo & 30 & $25 \%$ & 11 & $8 \%$ & 41 & $<0,001$ \\
\hline Negativo & 4 & $3 \%$ & 8 & $5 \%$ & 12 & \\
\hline Não realizado & 87 & $72 \%$ & 127 & $87 \%$ & 214 & \\
\hline Infarto Antigo & 14 & $12 \%$ & 3 & $2 \%$ & 17 & 0,004 \\
\hline Revascularização & 14 & $12 \%$ & 4 & $3 \%$ & 18 & 0,009 \\
\hline Hipertensão Arterial Sistêmica & 88 & $73 \%$ & 89 & $61 \%$ & 177 & 0,058 \\
\hline Diabetes Melito & 34 & $28 \%$ & 33 & $23 \%$ & 67 & 0,374 \\
\hline Dislipidemia & 80 & $66 \%$ & 89 & $61 \%$ & 169 & 0,458 \\
\hline Antecedentes Familiares & 50 & $41 \%$ & 78 & $53 \%$ & 128 & 0,065 \\
\hline \multicolumn{7}{|l|}{$\underline{\text { Atividade Física }}$} \\
\hline Ativo & 51 & $42 \%$ & 64 & $44 \%$ & 115 & 0,903 \\
\hline Atleta & 1 & $1 \%$ & 1 & $1 \%$ & 2 & \\
\hline Sedentário & 69 & $57 \%$ & 81 & $55 \%$ & 150 & \\
\hline \multicolumn{7}{|l|}{$\underline{\text { Etilismo }}$} \\
\hline Alcoólatra & 2 & $2 \%$ & 1 & $1 \%$ & 3 & 0,415 \\
\hline Bebe socialmente & 54 & $45 \%$ & 76 & $52 \%$ & 130 & \\
\hline Não bebe & 65 & $54 \%$ & 69 & $47 \%$ & 134 & \\
\hline
\end{tabular}

* Ecocardiografia sob Estresse pelo Esforço Físico (EEEF).

Fonte: Tabela elaborada pelo autor.

\subsection{Gravidade da DPOC (GOLD)}

Foi demonstrada no grupo G1 a classificação da DPOC com critérios do GOLD, apresentando a maior parte da população com DPOC leve (77 indivíduos) representando 63,6\%. 
Tabela 7 - Determinação da gravidade da DPOC através do GOLD (G1 n=121).

\begin{tabular}{lr}
\hline GOLD & N - G1* (\%) \\
\hline 1 - Leve & $77(63,6 \%)$ \\
2 - Moderado & $29(23,9 \%)$ \\
3 - Grave & $14(11,5 \%)$ \\
$4-$ Muito Grave & $0(0 \%)$ \\
\hline
\end{tabular}

* Resultados somente do grupo com DPOC (G1).

Fonte: Tabela elaborada pelo autor.

\subsection{Regressão logística}

Foi realizada a regressão logística para verificar a associação dos fatores de risco para isquemia miocárdica, com resultado de associação com valor significativo apenas para DPOC e Diabetes Melito (DM).

Tabela 8 - Regressão Logística para fatores de risco de isquemia miocárdica

\begin{tabular}{lrrrr}
\hline & Coeficiente & Erro padrão & Valor z & Valor p \\
\hline DPOC & $\mathbf{1 , 4 7 7}$ & $\mathbf{0 , 2 8 8}$ & $\mathbf{5 , 1 3 2}$ & $<\mathbf{0 , 0 0 1}$ \\
Tabagismo & 0,004 & 0,279 & 0,015 & 0,988 \\
Idade & 0,014 & 0,013 & 1,091 & 0,275 \\
Diabetes melito & $\mathbf{0 , 5 8 8}$ & $\mathbf{0 , 2 7 1}$ & $\mathbf{2 , 1 6 9}$ & $\mathbf{0 , 0 3 0}$ \\
Antecedentes familiares & 0,287 & 0,243 & 1,184 & 0,236 \\
Hipertensão arterial sistêmica & 0,398 & 0,255 & 1,561 & 0,119 \\
IMC* & $-0,024$ & 0,027 & $-0,876$ & 0,381 \\
Depressão & 0,356 & 0,278 & 1,281 & 0,200 \\
Dislipidemia & 0,431 & 0,245 & 1,759 & 0,079 \\
Etilismo & 0,171 & 0,229 & 0,749 & 0,454 \\
Atividade física & 0,132 & 0,229 & 0,575 & 0,565 \\
\hline
\end{tabular}

* Índice de massa corpórea (IMC).

Fonte: Tabela elaborada pelo autor. 


\section{DISCUSSÃO}

Dos 267 tabagistas avaliados, a isquemia miocárdica foi estatisticamente significativa no grupo dos portadores da DPOC, sendo encontrada em $61 \%$ desses, fato extremamente relevante quando comparamos com Patel et al. (2012), que realizou uma coorte com 386 pacientes, e encontrou apenas $16 \%$ de isquemia miocárdica, em indivíduos com DPOC, embora sua indicação tenha sido a busca de isquemia miocárdica em portadores de DPOC, diferentemente desta pesquisa onde o foco eram indivíduos com suspeita isquemia miocárdica.

A prevalência de isquemia miocárdica em indivíduos com DPOC é variável na literatura, como, por exemplo, o trabalho de Smith e Wrobel (2014), que encontrou prevalência de isquemia em $15 \%$ da população com DPOC. Justifica-se, entre outros fatores, os hábitos de vida de cada população envolvida, principalmente quando relacionamos com o vício do tabaco. A idade avançada, o tabagismo, juntamente com a alta prevalência de hipertensão arterial sistêmica em pacientes com DPOC, dados encontrados também nesta pesquisa, são fatores que aumentam o risco de isquemia miocárdica. Apesar do grupo com DPOC ser mais idoso, a regressão logística revelou que a isquemia miocárdica foi independente de todos esses fatores (AGUSTÍ et al., 2012).

Outros fatores associados como o estresse oxidativo, o aumento da ativação plaquetária e os processos inflamatórios sistêmicos, incluindo o aumento circulatório de citocinas: o fator de necrose tumoral (TNF- $\alpha$ ), a interleucina 6 (IL-6), a proteína $\mathrm{C}$ reativa (PCR) e o fibrinogênio, ocorrem em pacientes com DPOC, principalmente nos períodos de exacerbação, e também estão presentes em outras condições inflamatórias, tais como a aterosclerose, o que justifica os achados desta pesquisa, pois a presença de isquemia miocárdica foi independente desses fatores e da gravidade da DPOC (DÍEZ; MORGAN; GARCÍA, 2013).

O mecanismo fisiopatológico que a DPOC e a doença cardiovascular têm em comum é a hipoxemia, que ocorre com maior regularidade em indivíduos com DPOC classificada como grave, podendo ainda ser precipitada por atividades físicas, apneia do sono ou ainda quando existe tosse em excesso. Outros fatores que estão presentes na DPOC são a obesidade e o sedentarismo, os quais também são fatores que estão associados à isquemia miocárdica, embora não consoantes com os resultados obtidos nesta amostra (SCHNEIDER et al., 2010; WATZ et al., 2009). 
Outras evidências colocam a hiperinsuflação do tórax do paciente com DPOC como fator que pode diminuir o fluxo sanguíneo que chega ao coração (pré-carga), aumentando o risco para hipóxia, podendo levar a uma isquemia. Porém, na atual pesquisa os indivíduos estão na maior parte dentro classificação da DPOC leve $(63,6 \%)$ e, mesmo sendo pouco sintomáticos, tiveram uma alta prevalência de isquemia miocárdica (BORGES; VIANNA; TERRA FILHO, 2003; SCLAUSER PESSOA et al., 2012).

Não existiu diferença com relação a sexo, cor observada e nível de ensino, o que pode ser justificado pela prevalência da DPOC, que no mundo varia consideravelmente pelas diferenças nos hábitos tabágicos, sem considerar outros fatores sociodemográficos. Em alguns estudos o sexo masculino e a idade foram marcados como fatores de risco para DPOC, porém quando é realizada uma análise controlada para resultados de tabaco, história ocupacional e outros fatores de confusão, o risco para homens e mulheres torna-se igual (BÁRBARA et al., 2013).

Em outro estudo, com 294 pacientes, 151 (51,4\%) eram homens e 143 (48,6\%) eram mulheres. Dos homens, 110 (72,8\%) tiveram DPOC, em comparação com 75 (52,5\%) entre as mulheres, devido aos homens estarem mais expostos aos fatores de risco (LOGANATHAN et al., 2006).

Ratificando a literatura, o tabagismo se mostrou fator predominante para a presença da DPOC, tanto na maior carga tabágica, como na manutenção desta atividade, sendo o fator primordial para a ocorrência da DPOC. Pois se sabe que o tabagismo é o principal fator de risco para a DPOC (KISSMANN; LEAL; FRANCO, 2013; SCHNEIDERS et al., 2015).

O grupo G1 apresentou maior frequência de tosse crônica e relato de expectoração, resultados esperados, pois fazem parte dos sintomas crônicos que contribuem para o diagnóstico da DPOC. Apesar dos participantes da pesquisa terem apresentado maior frequência no mMRC nível 1, que demonstra dispneia ao esforço leve, revelando assim o paciente pouco sintomático para DPOC (ROCHA; PEREIRA, 2009; VOLL-AANERUD et al., 2008).

As comorbidades como: osteoporose, depressão e neoplasias foram mais frequentes no grupo com DPOC, dados encontrados em outras pesquisas revelam que essas são as comorbidades com maior impacto na DPOC. A presença de osteoporose nestes pacientes pode estar ligada ao fato das citocinas pró-inflamatórias alterarem o metabolismo ósseo, podendo aumentar o nível de osteoclastos, aumentando assim a reabsorção óssea. Outros fatores de risco podem estar associados com a osteoporose e a DPOC, e esses fatores podem estar ligados ao 
tabagismo, à deficiência de vitamina D e ao uso de corticosteroides. (CAZZOLA et al., 2010; DUCKERS et al., 2010).

Uma das teorias para explicar a depressão associada à DPOC está na piora na qualidade de vida, podendo levar à incapacitação, ou ainda quando existe aumento da autopercepção dos sintomas da doença e isolamento social, compondo um ciclo vicioso da piora da qualidade de vida do indivíduo, embora seja destoante nesta amostra, pois os portadores da DPOC eram poucos sintomáticos e com capacidade física preservada, tanto que puderam ser submetidos à EEEF (ARAUJO; ARAUJO, 2013; GODOY; GODOY, 2002).

A associação entre depressão e a isquemia miocárdica está ligada a diversos fatores psicológicos e fisiopatológicos, dentre os quais vale destacar: os fatores imunológicos, o aumento da atividade plaquetária, a variação da frequência cardíaca e principalmente a aterogênese inflamatória, fator comum com a DPOC, porém neste trabalho foi realizada a regressão logística, a qual demonstrou que não existiu associação da isquemia miocárdica com a depressão (OLIVEIRA et al., 2015).

Outro fator envolvido é o conhecimento de que a Diabetes Melito (DM) é associada à isquemia miocárdica, pois devido à hipoglicemia grave normalmente ocorre a liberação de catecolaminas que promovem a vasoconstricção, a agregação plaquetária e, consequentemente, fenômenos isquêmicos. Esta associação foi observada neste estudo, entretanto, não houve diferença significativa quanto a DM entre os grupos G1 e G2, mesmo sabendo que a DM é uma das comorbidades mais prevalentes da DPOC (DE LUCCIA, 2003).

Ademais, a inflamação crônica que ocorre na DPOC desempenha um papel importante e significativo no aparecimento de neoplasias. A inflamação da via aérea, resultante da exposição à fumaça do tabaco, inflama a mucosa brônquica, causando danos na célula epitelial, o que provoca sua rápida substituição, podendo levar a erros no DNA celular, ampliando o processo de carcinogênese (MALKINSON, 2004; ZAMBONI, 2013).

Quanto aos sintomas cardiovasculares, o grupo G1 apresentou maior frequência de precordialgia típica e atípica do que o grupo G2, já que os achados de isquemia miocárdica no G1 foram mais frequentes, tendo em vista que as precordialgias são sintomas clínicos do paciente predisposto a ter isquemia miocárdica (DIAMOND; FORRESTER, 1979; VACANTI; SESPEDES; SARPI, 2004). 
O grupo G1 obteve resultados de maior frequência com relação a: cateterismo positivo, infartos antigos e revascularização do miocárdio pregressa, possivelmente explicados pela alta prevalência da doença cardiovascular na DPOC (FABBRI; RABE, 2007; SCHETTINO et al., 2013).

\section{CONCLUSÃO}

1. A DPOC é um fator presente em indivíduos com suspeita de isquemia, submetidos à EEEF, mesmo nos oligossintomáticos de fase inicial da doença obstrutiva;

2. O grupo da DPOC mostrou média significativamente maior nas áreas de isquemia;

3. A DPOC foi diagnosticada em $45 \%$ dos tabagistas com suspeita de isquemia miocárdica submetidos à EEEF;

4. As comorbidades de maior impacto na DPOC: osteoporose, depressão, neoplasias e doença isquêmica, foram as estatisticamente significantes nesta pesquisa. 


\section{REFERÊNCIAS BIBLIOGRÁFICAS}

ADELOYE, D. et al. Global and regional estimates of COPD prevalence: Systematic review and meta-analysis. Journal of Global Health, v. 5, n. 2, p. 20415, 2015.

AGUSTÍ, A. et al. Persistent systemic inflammation is associated with poor clinical outcomes in copd: A novel phenotype. PLoS ONE, v. 7, n. 5, 2012.

ALEXOPOULOS, E. C. et al. Frequency and risk factors of COPD exacerbations and hospitalizations: A nationwide study in greece (Greek obstructive lung disease epidemiology and health ecoNomics: GOLDEN study). International Journal of COPD, v. 10, n. 1, p. 26652674, 2015.

ANDRADE, J. et al. II Diretrizes da Sociedade Brasileira de Cardiologia Sobre Teste Ergométrico. Arquivos Brasileiros de Cardiologia, v. 78, 2002.

ARAujo, M. S.; ARAujO, J. S. DPOC e Depressão. Pulmão RJ, Rio de Janeiro, v. 22, n. 2, p. 35-39, 2013.

BÁRBARA, C. et al. Chronic obstructive pulmonary disease prevalence in lisbon, portugal: The burden of obstructive lung disease study. Revista Portuguesa de Pneumologia, v. 19, n. 3, p. 96-105, 2013.

BARNES, P. J.; CELLI, B. R. Systemic manifestations and comorbidities of COPD. European Respiratory Journal, v. 33, n. 5, p. 1165-1185, 2009.

BHANDARI, R.; SHARMA, R. Epidemiology of chronic obstructive pulmonary disease: a descriptive study in the mid-western region of Nepal. International journal of chronic obstructive pulmonary disease, v. 7, p. 253-7, 2012.

BOCCHI, E. A. et al. III Brazilian Guidelines on Chronic Heart Failure. Arquivos brasileiros de cardiologia, v. 93, n. 1 Suppl 1, p. 3-70, 2009.

BORGES, M. C.; VIANNA, E. S. O.; TERRA FILHO, J. Abordagem terapêutica na exacerbação da doença pulmonar obstrutiva crônica (DPOC). Medicina, v. 36, n. 2-4, p. 241-247, 2003.

CAMPOS FILHO, O. et al. Diretriz para Indicações e utilização da ecocardiografia na prática clínica. Arquivos Brasileiros de Cardiologia, v. 82, p. 11-34, 2004.

CAZZOLA, M. et al. Prevalence of comorbidities in patients with chronic obstructive pulmonary disease. Respiration, v. 80, n. 2, p. 112-119, 2010.

CÉSAR, L. A. M. et al. Diretrizes de doença coronariana crônica e angina estável. Arquivos Brasileiros de Cardiologia, v. 83, p. 2-43, 2004.

DE LUCCIA, N. Doença vascular e diabetes. J Vasc Br, v. 2, n. 1, p. 49-60, 2003. 
DIAMOND, G. A.; FORRESTER, J. S. Analysis of probability as an aid in the clinical diagnosis of coronary-artery disease. The new england journal of medicine, p. 9, 1979.

DIAZ-GUZMAN, E.; MANNINO, D. M. Epidemiology and prevalence of chronic obstructive pulmonary disease. Clinics in Chest Medicine, v. 35, n. 1, p. 7-16, 2014.

DÍEZ, J. DE M.; MORGAN, J. C.; GARCÍA, R. J. The association between COPD and heart failure risk: a review. International journal of chronic obstructive pulmonary disease, v. $8, \mathrm{p}$. 305-12, 2013.

DIVO, M. et al. Comorbidities and risk of mortality in patients with chronic obstructive pulmonary disease. American Journal of Respiratory and Critical Care Medicine, v. 186, n. 2, p. 155-161, 2012.

DUCKERS, J. M. et al. Cardiovascular and musculskeletal co-morbidities in patients with alpha 1 antitrypsin deficiency. Respiratory research, v. 11, n. 1, p. 173, 2010.

DUSSER, D. et al. Differences in outcomes between GOLD groups in patients with COPD in the TIOSPIR??trial. International Journal of COPD, v. 11, p. 133-145, 2016.

FABBRI, L. M.; RABE, K. F. From COPD to chronic systemic infl ammatory syndrome? Lancet, v. 370, p. 797-799, 2007.

FEARY, J. R. et al. Prevalence of major comorbidities in subjects with COPD and incidence of myocardial infarction and stroke: a comprehensive analysis using data from primary care. Thorax, v. 65, n. 11, p. 956-962, 2010.

GARDIN, J. M. et al. Recommendations for a standardized report for adult transthoracic echocardiography: A report from the American Society of Echocardiography's Nomenclature and Standards Committee and Task Force for a Standardized Echocardiography report. Journal of the American Society of Echocardiography, v. 15, n. 3, p. 275-290, 2002.

GODOY, D. V.; GODOY, R. F. Redução nos níveis de ansiedade e depressão de pacientes com doença pulmonar obstrutiva crônica (DPOC) participantes de um programa de reabilitação pulmonar. Jornal Pneumologia, v. 28, n. 54, p. 120-124, 2002.

GOLD. Global Strategy for the Diagnosis, Management, and Prevention of Chronic Obstructive Lung Disease 2017 Report. American journal of respiratory and critical care medicine, p. 174, $2017 \mathrm{a}$.

GOLD, G. I. FOR C. O. L. Global Initiative for Chronic Obstructive Lung. Global Initiative for Chronic Obstructive Lung Disease website, 2017 b.

GUPTA, R.; MANN, S. Correlation Between COPD And Echocardiographic Features With Severity Of Disease. v. 7, n. 1, 2016. 
HAGSTAD, S. et al. COPD among non-smokers - Report from the Obstructive Lung Disease in Northern Sweden (OLIN) studies. Respiratory Medicine, v. 106, n. 7, p. 980-988, 2012.

HAN, M. K. et al. Chronic obstructive pulmonary disease phenotypes: The future of COPD. American Journal of Respiratory and Critical Care Medicine, v. 182, n. 5, p. 598-604, 2010.

HASAN, A. et al. Understanding the relation between COPD and coronary artery disease. Journal, Indian Academy of Clinical Medicine, v. 15, n. 2, p. 120-124, 2014.

JONES, P. W. et al. Development and first validation of the COPD Assessment Test. European Respiratory Journal, v. 34, n. 3, p. 648-654, 2009.

KISSMANN, G.; LEAL, R.; FRANCO, C. Apneia obstrutiva do sono: da suspeita diagnóstica ao tratamento. J. bras. med, v. 101, 2013.

KOVELIS, D. et al. Validação do Modified Pulmonary Functional Status and Dyspnea Questionnaire e da escala do Medical Research Council para o uso em pacientes com doença pulmonar obstrutiva crônica no Brasil. Jornal Brasileiro de Pneumologia, v. 34, n. 12, p. 10081018, 2008.

LANG, R. M. et al. Recommendations for cardiac chamber quantification by echocardiography in adults: An update from the American society of echocardiography and the European association of cardiovascular imaging. European Heart Journal Cardiovascular Imaging, v. 16, n. 3, p. 233-271, 2015.

LINS, R.; ESTEVES, J.; VIDIGAL, M. Avaliação da Qualidade de Vida e da Funcionalidade de um paciente com DPOC grave antes e após Reabilitação Cardiopulmonar e Metabólica domiciliar : Relato de caso Resumo Introdução. v. 3, n. 1, p. 57-64, 2012.

LOGANATHAN, R. S. et al. Prevalence of COPD in Women Compared to Men Around the Time of Diagnosis of Primary Lung Cancer. Chest, v. 129, n. 5, p. 1305-1312, 2006.

LOPEZ, A. D. et al. Chronic obstructive pulmonary disease: Current burden and future projections. European Respiratory Journal, v. 27, n. 2, p. 397-412, 2006.

LWANGA, S.; LEMESHOW, S. Sample size determination in health studies: A practical manual, 1991. World Health Organization, Geneva, p. 88, 1991.

MACDONALD, M. I. et al. Cardiac dysfunction during exacerbations of chronic obstructive pulmonary disease. The Lancet Respiratory Medicine, v. 4, n. 2, p. 138-148, 2016.

MAHBOUB, B. et al. Comorbidities associated with COPD in the middle East and North Africa region: Association with severity and exacerbations. International Journal of COPD, v. 11, p. 273-280, 2016. 
MALKINSON, A. M. Role Of Inflammation In Mouse Lung Tumorigenesis: A Review. Experimental Lung Research, v. 31, n. 1, p. 57-82, 2004.

MENEZES, A. M. B. PLATINO - Projeto Latino Americano de Investigação em Obstrução Pulmonar. p. 1-88, 2006.

MESQUITA, E. T.; JORGE, A. J. L. Entendendo a disfunção diastólica assintomática na prática clínica. Arquivos Brasileiros de Cardiologia, v. 100, n. 1, p. 94-101, 2013.

MS, M. DA S. VIGITEL 2014: Vigilância de fatores de Risco para doenças crônicas por inquérito telefônico. [s.l: s.n.].

NAKAJIMA, K. et al. A possible association between suspected restrictive pattern as assessed by ordinary pulmonary function test and the metabolic syndrome. Chest, v. 134, n. 4, p. $712-$ $718,2008$.

NEVES, M. C. L. C. et al. Avaliação de atopia em portadores de DPOC. J Bras Pneumol, v. 39, n. 3, p. 323-328, 2013.

OLIVEIRA, N. A. et al. Mecanismos fisiopatológicos da associação entre depressão e síndrome coronariana aguda. Revista debates em psiquiatria, v. 4, n. 5, p. 48, 2015.

ONGEL, E. A. KKUTUK et al. How do COPD comorbidities affect ICU outcomes? International journal of chronic obstructive pulmonary disease, v. 9, p. 1187-1196, 2014.

PATEL, A. R. C. et al. The impact of ischemic heart disease on symptoms, health status, and exacerbations in patients with COPD. Chest, v. 141, n. 4, p. 851-857, 2012.

PEREIRA, C. A. D. C. Espirometria. Jornal Brasileiro de Pneumologia, v. 28, n. supl 3, p. S1S82, 2002.

PESSÔA, C. L. C.; PESSÔA, R. S. Epidemiologia da DPOC no presente - aspectos nacionais e internacionais. Pulmão RJ, Rio de Janeiro, v. 1, n. 1, p. 7-12, 2009.

PRICE, D. et al. Earlier diagnosis and earlier treatment of COPD in primary care. Primary Care Respiratory Journal, v. 20, n. 1, p. 15-22, 2011.

ROCHA, C. L. A. C. DA; PEREIRA, C. A. DE C. Dispneia em DPOC: Além da escala modified Medical Research Council *. J Bras Pneumol, v. 2, n. 2, p. 03-11, 2009.

SCHETTINO, C. D. S. et al. Relação entre DPOC e Doença Cardiovascular. Pulmão RJ, Rio de Janeiro, v. 50, p. 94, 2013.

SCHNEIDER, C. et al. Chronic obstructive pulmonary disease and the risk of cardiovascular diseases. European Journal of Epidemiology, v. 25, n. 4, p. 253-260, 2010. 
SCHNEIDERS, P. D. B. et al. Perfil tabágico e clínico dos portadores de doença pulmonar obstrutiva crônica que participam de pesquisa clínica em Santa Cruz do Sul - RS. Rev Epidemiol Control Infect., v. 5, n. 1, p. 37-41, 2015.

SCLAUSER PESSOA, I. M. B. et al. Efeitos da ventilação não-invasiva sobre a hiperinsuflação dinâmica de pacientes com DPOC durante atividade da vida diária com os membros superiores. Brazilian Journal of Physical Therapy, v. 16, n. 1, p. 61-67, 2012.

SHAVELLE, R. M. et al. Life expectancy and years of life lost in chronic obstructive pulmonary disease: findings from the NHANES III Follow-up Study. International journal of chronic obstructive pulmonary disease, v. 4, p. 137-148, 2009.

SILVA, S. T. DA et al. Combate ao Tabagismo no Brasil: a importância estratégica das ações governamentais. Ciência \& Saúde Coletiva, v. 19, n. 2, p. 539-552, 2014.

SMITH, M. C.; WROBEL, J. P. Epidemiology and clinical impact of major comorbidities in patients with COPD. Int J Chron Obstruct Pulmon Dis, v. 9, p. 871-888, 2014.

SORIANO, J. B. et al. Patterns of Comorbidities in Newly Diagnosed COPD and Asthma in Primary Care. Chest, p. 2099-2107, 2005.

STÄLLBERG, B. et al. The prevalence of comorbidities in COPD patients, and their impact on health status and COPD symptoms in primary care patients: a protocol for an UNLOCK study from the IPCRG. npj Primary Care Respiratory Medicine, v. 26, n. August, p. 16069, 2016.

VACANTI, L. J.; SESPEDES, L. B. H.; SARPI, M. D. O. O teste ergométrico é útil, seguro e eficaz, mesmo em indivíduos muito idosos, com 75 anos ou mais. Arquivos Brasileiros de Cardiologia, v. 82, n. $\mathrm{n}^{\mathrm{O}} 2$ 2, p. 147-150, 2004.

VOLL-AANERUD, M. et al. Respiratory symptoms, COPD severity, and health related quality of life in a general population sample. Respiratory Medicine, v. 102, n. 3, p. 399-406, 2008.

WATZ, H. et al. Physical activity in patients with COPD. European Respiratory Journal, v. 33 , n. 2, p. 262-272, 2009.

WORLD HEALTH ORGANIZATION. World Health statistics 2014. [s.l: s.n.].

WÜNSCH FILHO, V. et al. Tabagismo e câncer no Brasil: evidências e perspectivas. Revista Brasileira de Epidemiologia, v. 13, n. 2, p. 175-187, 2010.

ZAMBONI, M. Câncer do Pulmão e DPOC. Pulmão RJ, Rio de Janeiro, v. 22, n. 2, p. 40-44, 2013. 


\section{APÊNDICE \\ TERMO DE CONSENTIMENTO LIVRE E ESCLARECIDO}

Resolução nº 466, de 13 de junho de 2013, Diário Oficial da União, sendo o Conselho Nacional de Saúde (CNS).

O presente termo em atendimento às normas éticas exigidas pela Resolução 466/2012 (Conselho Nacional de Saúde), destina-se a esclarecer ao participante da pesquisa intitulada "Análise Comparativa de pacientes tabagistas com e sem DPOC com suspeita de isquemia miocárdica", sob responsabilidade dos pesquisadores Dra. Maria Luiza Dória Almeida e Marcos Gabriel do Nascimento Junior do curso de Mestrado em Ciências da Saúde da Universidade Federal de Sergipe, os seguintes aspectos:

Objetivo: Avaliar DPOC em pacientes com suspeita de disfunção ventricular esquerda.

Justificativa e Relevância: A Doença Pulmonar Obstrutiva Crônica (DPOC) e as Cardiopatias são as principais causas de apresentação aos cuidados primários e secundários nos Serviços de Saúde. O comprometimento cardiovascular é a principal comorbidade da DPOC e a disfunção diastólica é um marcador de dano orgânico e está associada à grande incidência de eventos cardiovasculares. Há lacuna no conhecimento entre a disfunção diastólica em tabagista com DPOC e seu real valor na insuficiência cardíaca e este projeto pretende contribuir com o tema, avaliando os DPOC com suspeita de disfunção ventricular esquerda. Existe a necessidade de pesquisas baseadas em evidencias científicas sobre a função ventricular em doentes com DPOC, a fim da realização de diagnósticos precisos na tentativa de prevenção e gestão de eventos adversos. Em vista disso, este trabalho questiona-se como se comporta a função ventricular de pacientes com DPOC.

Participação: Nesse estudo você será submetido a uma entrevista com a aplicação de um roteiro semiestruturado contendo informações identificadoras correspondentes aos instrumentos da pesquisa.

Desconfortos e riscos: este estudo não causa nenhum tipo de riscos direto aos participantes, podendo haver apenas algum desconforto quanto às perguntas diretas do instrumento de coleta.

Confidencialidade do estudo: será assegurado sigilo para todos os participantes da pesquisa, o nome da pessoa não será identificado no instrumento de coleta. 
Benefícios: esta pesquisa poderá criar subsídios para implementação de novas técnicas de diagnóstico para a Doença Pulmonar Obstrutiva Crônica e a disfunção ventricular.

Dano advindo da pesquisa: esta pesquisa não oferece nenhum dano aos participantes.

Garantia de esclarecimento: são garantidos aos participantes da pesquisa esclarecimentos adicionais em qualquer momento da pesquisa.

Participação Voluntária: a participação dos profissionais nessa pesquisa é voluntária e livre de qualquer forma de remuneração.

Consentimento para participação: Eu estou de acordo com a participação no estudo descrito acima. Eu fui devidamente esclarecido quanto os objetivos da pesquisa, aos procedimentos aos quais serei submetido e os possíveis riscos envolvidos na minha participação. Os pesquisadores me garantiram disponibilizar qualquer esclarecimento adicional a que eu venha solicitar durante o curso da pesquisa e o direito de desistir da participação em qualquer momento, sem que a minha desistência implique em qualquer prejuízo à minha pessoa ou à minha família, sendo garantido anonimato e o sigilo dos dados referentes à minha identificação, bem como de que a minha participação neste estudo não me trará nenhum benefício econômico.

Eu, aceito livremente participar do estudo intitulado "Análise Comparativa de pacientes tabagistas com e sem DPOC com suspeita de isquemia miocárdica" desenvolvido pelo mestrando Marcos Gabriel do Nascimento Junior, sob a responsabilidade da Professora Dra. Maria Luiza Dória Almeida da Universidade Federal de Sergipe (UFS).

Nome da Participante

Nome da pessoa ou responsável legal

\section{COMPROMISSO DO PESQUISADOR}

Eu discuti as questões acima apresentadas com cada participante do estudo. É minha opinião que cada indivíduo entenda os riscos, benefícios e obrigações relacionadas a esta pesquisa.

Aracaju-SE, Data:

Assinatura do Pesquisador

Para maiores informações, pode entrar em contato com:

Marcos Gabriel do Nascimento Junior. Fone: (79) 9900-7088

Dra. Maria Luiza Dória Almeida. Fone: (79) 9988-8683 
ANEXOS 


\section{ANEXO A}

\section{SEDAR (Serviço de doença do aparelho respiratório) QUESTIONÁRIO - Avaliação Clínica}

Responsáveis: Igor Larchert Mota; Marcos Gabriel do Nascimento Junior; Maria Luiza Doria Almeida.

Nome Sexo(Mas1,Fem2)

Data/Nascimento

Altura (cm) Peso

(Kg) Escolaridade

(anos estudados) Cor observada (1,branca 2,negra, 3parda, 4 oriental)

Ocupação Atual: Ocupação Anterior Renda familiar

(salário mínimo)

Responda (sim 1 ou não 2) Fuma $\square$ ex-fumante $\square$ fumou q ${ }^{\text {to }}$ tempo $\square$ maço/an $\square$ Exposição len

Risque o numero referido pelo paciente na pergunta para (COPD Assessment Test) CAT

\begin{tabular}{|c|c|c|c|c|c|c|c|}
\hline 1. Nunca tenho tosse & 0 & 1 & 2 & 3 & 4 & 5 & Tenho tosse o tempo todo \\
\hline 2. Não tenho nenhum catarro (secreção) no peito & 0 & 1 & 2 & 3 & 4 & 5 & O meu peito está cheio de catarro (secreção) \\
\hline 3. Não sinto nenhuma pressão no peito & 0 & 1 & 2 & 3 & 4 & 5 & Sinto uma grande pressão no peito \\
\hline $\begin{array}{l}\text { 4. Não sinto falta de ar quando subo uma ladeira ou UM } \\
\text { lance de escada }\end{array}$ & 0 & 1 & 2 & 3 & 4 & 5 & $\begin{array}{l}\text { Sinto bastante falta de ar quando subo uma ladeira ou UM } \\
\text { andar de escada }\end{array}$ \\
\hline $\begin{array}{l}\text { 5. Não sinto nenhuma limitação nas minhas atividades em } \\
\text { casa }\end{array}$ & 0 & 1 & 2 & 3 & 4 & 5 & Sinto-me muito limitado nas minhas atividades em casa \\
\hline $\begin{array}{l}\text { 6. Sinto-me confiante para sair de casa, apesar da minha DP } \\
\text { doença pulmonar. }\end{array}$ & 0 & 1 & 2 & 3 & 4 & 5 & $\begin{array}{l}\text { Não me sinto nada confiante para sair de casa, por causa da } \\
\text { minha doença pulmonar. }\end{array}$ \\
\hline 7. Dorme profundamente & 0 & 1 & 2 & 3 & 4 & 5 & Não dorme profundamente devido à minha doença pulmonar \\
\hline 8. Tenho muita energia (disposição) & 0 & 1 & 2 & 3 & 4 & 5 & Não tenho nenhuma energia (disposição) \\
\hline
\end{tabular}

Escala de dispneia do Medical Research Council (MRC): (0.Dispneia a exercícios intensos. ; 1. Dispneia andando rápido no plano ou subindo aclives leves. 2. Andar mais lentamente que pessoas da mesma idade devido à dispneia ou parar para respirar andando normalmente no plano. 3. Parar para respirar após caminhar 90 metros ou alguns minutos no plano. 4. Não sai de casa devido à dispneia ou precisa de ajuda para trocar de roupa.

Questionário de vias aéreas 20 (AQ20) Responda (sim 1 ou não 2) Você

\begin{tabular}{|c|c|}
\hline 01. Tem crises de tosse durante o dia? & $\begin{array}{l}\text { 11. Sente falta de ar para as suas atividades durante o } \\
\text { trabalho? }\end{array}$ \\
\hline 03. Sente falta de ar ao cuidar do jardim devido a sua DP? & 13. Devido a sua DP sente falta de ar $\mathrm{n}$ as tarefas domésticas? \\
\hline $\begin{array}{l}\text { 04. Se preocuparia em ir à casa de um amigo se lá existisse algo que pudesse causar } \\
\text { uma crise de sintomas pulmonares? }\end{array}$ & $\begin{array}{l}\text { 14. Devido a DP tem q voltar pra casa mais cedo do que as } \\
\text { outras pessoas após um programa noturno? }\end{array}$ \\
\hline $\begin{array}{l}\text { 05. Tem sintomas pulmonares quando exposto a cheiros fortes, cigarro ou } \\
\text { perfume? }\end{array}$ & 15. Tem falta de ar quando está rindo devido a sua DP? \\
\hline 07. Fica com falta de ar enquanto tenta dormir? & $\begin{array}{l}\text { 17. Devido a sua DP você sente que não consegue aproveitar } \\
\text { a vida? }\end{array}$ \\
\hline $\begin{array}{l}\text { 08. Você fica preocupado com os efeitos em longo prazo na sua saúde causados } \\
\text { REMÉDIOS que você tem que tomar por causa da sua doença pulmonar? }\end{array}$ & $\begin{array}{l}\text { 18. Devido a sua DP você sente muito enfraquecido após } \\
\text { resfriado? }\end{array}$ \\
\hline 09. Os seus sintomas pulmonares pioram quando você fica aborrecido? & 19. Você tem a sensação constante de um peso no tórax? \\
\hline
\end{tabular}




\title{
Comorbidade
}

Quantas "gripes" ou piora (exacerbação) no ultimo ano

$\square$

foi hospitalizado ( $\operatorname{sim} \mathbf{1}$ ou não 2$)$

Você tem alguma doença ( $\operatorname{sim} \mathbf{1}$ ou não 2$)$ ?Cardiopatia outras osteoporose depressão $\mathrm{q}^{\text {tos }} \operatorname{dias}$

Você perdeu peso (( $\operatorname{sim} \mathbf{1}$ ou não 2$)$ ?

Quantos kg?

Tratamento

Você toma remédio para DPOC (bronquite, enfisema) ( $\operatorname{sim} 1$ ou não 2$)$ ? SABA LAMA

XANTINA $\square$

ACETILCISTEINA

DAXAS

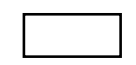

CS corticoide sistêmico

SAMA $\square$ LABA câncer $\square$

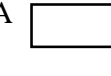

\begin{abstract}
DAXAS
\end{abstract}
CI corticoide inalatório 


\begin{abstract}
ANEXO B
ESCALA DE DISPNÉIA Medical Research Council MODIFICADA
\end{abstract}

0. Tenho falta de ar durante exercícios intensos.

1. Tenho falta de ar quando andando apressadamente ou subindo uma rampa leve.

2. Preciso andar mais devagar do que pessoas da minha idade, ou preciso parar para respirar mesmo quando andando devagar.

3. Paro para respirar depois de andar menos de 100 metros ou após alguns minutos.

4. Sinto tanta falta de ar que não saio mais de casa, ou preciso de ajuda pra tomar banho ou me vestir sozinho.

Modificado de: (Kovelis D, Segretti NO, Probst VS, Lareau SC, Brunetto AF, Pitta F. Validation of the Modified Pulmonary Functional Status and Dyspnea Questionnaire and the Medical Research Council scale for use in Brazilian patients with chronic obstructive pulmonary disease. Jornal Brasileiro de Pneumologia. 2008 dez;34(12):1008-18). 


\begin{abstract}
ANEXO C
COPD Assessment Test - CAT

Versão traduzida para a língua portuguesa.
\end{abstract}

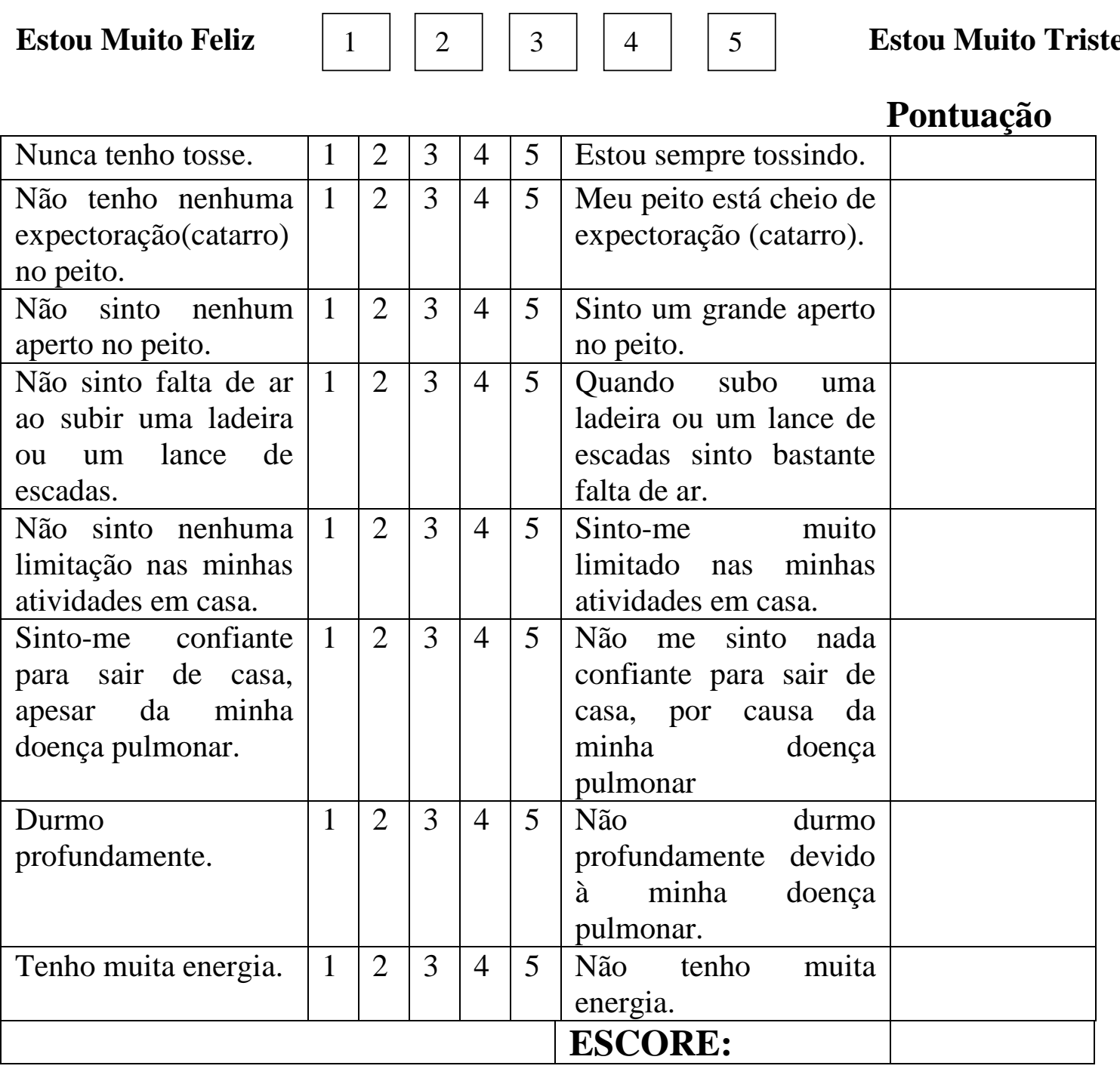

